\title{
Newsfeed Filtering and Dissemination for Behavioral Therapy on Social Network Addictions
}

\author{
Hong-Han Shuai \\ Department of Electrical and \\ Computer Engineering, National \\ Chiao Tung University, Taiwan \\ hhshuai@nctu.edu.tw \\ Yi-Feng Lan \\ Department of Social Work, Tunghai \\ University \\ Center for General Education and \\ Core Curriculum, Tamkang \\ University \\ carolyflan@gmail.com
}

\author{
Yen-Chieh Lien* \\ College of Information and Computer \\ Sciences \\ University of Massachusetts Amherst, \\ USA \\ ylien@umass.edu
}

Wang-Chien Lee

Department of Computer Science and Engineering, The Pennsylvania State

University, USA

wlee@cse.psu.edu

\author{
De-Nian Yang \\ Institute of Information \\ Science/Research Center for \\ Information Technology Innovation, \\ Academia Sinica, Taiwan \\ dnyang@iis.sinica.edu.tw \\ Philip S. Yu \\ Shanghai Institute for Advanced \\ Communication and Data Science, \\ Shanghai Key Laboratory of Data \\ Science, Fudan University \\ Computer Science Department, \\ University of Illinois at Chicago, USA \\ psyu@uic.edu
}

\begin{abstract}
While the popularity of online social network (OSN) apps continues to grow, little attention has been drawn to the increasing cases of Social Network Addictions (SNAs). In this paper, we argue that by mining OSN data in support of online intervention treatment, data scientists may assist mental healthcare professionals to alleviate the symptoms of users with SNA in early stages. Our idea, based on behavioral therapy, is to incrementally substitute highly addictive newsfeeds with safer, less addictive, and more supportive newsfeeds. To realize this idea, we propose a novel framework, called Newsfeed Substituting and Supporting System (N3S), for newsfeed filtering and dissemination in support of SNA interventions. New research challenges arise in 1) measuring the addictive degree of a newsfeed to an SNA patient, and 2) properly substituting addictive newsfeeds with safe ones based on psychological theories. To address these issues, we first propose the Additive Degree Model $(A D M)$ to measure the addictive degrees of newsfeeds to different users. We then formulate a new optimization problem aiming to maximize the efficacy of behavioral therapy without sacrificing user preferences. Accordingly, we design a randomized algorithm with a theoretical bound. A user study with 716 Facebook users and 11 mental healthcare professionals around the world manifests that the addictive scores can be reduced by more than $30 \%$. Moreover, experiments show that the correlation between the SNA scores and
\end{abstract}

*The work was done while the author was with Academia Sinica.

Permission to make digital or hard copies of all or part of this work for personal or classroom use is granted without fee provided that copies are not made or distributed for profit or commercial advantage and that copies bear this notice and the full citation on the first page. Copyrights for components of this work owned by others than ACM must be honored. Abstracting with credit is permitted. To copy otherwise, or republish, to post on servers or to redistribute to lists, requires prior specific permission and/or a fee. Request permissions from permissions@acm.org.

CIKM '18, October 22-26, 2018, Torino, Italy

(c) 2018 Association for Computing Machinery.

ACM ISBN 978-1-4503-6014-2/18/10 ..\$15.00

https://doi.org/10.1145/3269206.3271689 the addictive degrees quantified by the proposed model is much greater than that of state-of-the-art preference based models.

\section{CCS CONCEPTS}

- Human-centered computing $\rightarrow$ Social content sharing; • Computing methodologies $\rightarrow$ Ranking;

\section{KEYWORDS}

Social network analysis;online intervention;addiction

ACM Reference Format:

Hong-Han Shuai, Yen-Chieh Lien, De-Nian Yang, Yi-Feng Lan, Wang-Chien Lee, and Philip S. Yu. 2018. Newsfeed Filtering and Dissemination for Behavioral Therapy on Social Network Addictions. In The 27th ACM International Conference on Information and Knowledge Management (CIKM '18), October 22-26, 2018, Torino, Italy. ACM, New York, NY, USA, 10 pages. https://doi.org/10.1145/3269206.3271689

\section{INTRODUCTION}

While social media have inspired intensive research in recent years, few of them address the addiction problems arising along with the rise of online social networks (OSNs). Indeed, the number of users with Social Network Addictions (SNAs) significantly increases [26]. ${ }^{1}$ Facebook also admits that using Facebook could be harmful to users. ${ }^{2}$ A poll in CNN shows that $52 \%$ of teenagers are addicted to mobile OSNs. ${ }^{3}$ A recent study also indicates that SNAs may lead to excessive use, depression, social withdrawal, and negative repercussions [8]. Emotional states of SNA patients tend to deteriorate, causing hostility, compulsive behaviors, and a much higher risk of suicidal ideation [21]. Recently, Facebook Depression becomes a legitimate medical concern among adolescents [31]. SNAs are

\footnotetext{
${ }^{1}$ Notice that different clinical terms represent various levels of addictive substance use, e.g., social media abuse [17], social network dependence [35], social network addiction $[13,17,26]$. For simplicity, we use SNAs to represent them.

${ }^{2}$ https://www.cnbc.com/2017/12/15/facebook-just-admitted-that-using-facebookcan-be-bad-for-you.html

${ }^{3}$ http://edition.cnn.com/2016/05/03/health/teens-cell-phone-addiction-parents/
} 
so serious that some countries, e.g., South Korea, plan to regulate online social games by law, just like drugs and gambling. ${ }^{4}$

Detecting SNAs in early stages is crucial for timely clinical interventions. Standard questionnaires for quantifying addiction severity have been developed and validated $[10,13]$. However, it is not feasible to request every OSN user to take questionnaires regularly. We argue that the behaviors of users on OSNs may provide valuable signals for detecting SNAs early. However, mental healthcare professionals are not trained to collect and analyze data of user behaviors on OSNs (denoted as OSN data). In this paper, by teaming up with mental healthcare practitioners, we (data and computer scientists) propose novel data mining techniques to assist (1) identifying addictive contents to OSN users, and (2) facilitating online interventions for potential SNAs.

One naïve intervention treatment is total abstinence, i.e., prohibition of accessing OSNs by supervisors (parents, teachers, etc.). However, such a compulsive behavioral change usually results in relapse [26], i.e., revert to excessive usage after abstinence. Moreover, the tension between the patients and supervisors may lead to tragedies. ${ }^{5}$ Different from conventional intervention approaches, we analyze the addictiveness of OSN contents for individual patients in order to carry out "personalized" behavioral therapy [29] in early stages since different contents jointly affect the SNA scores of users. The main idea, similar to e-cigarettes, is to substitute highly addictive newsfeeds with informative and supportive contents based on behavioral therapy and social comparison theory. However, treating SNAs requires caution. Not only the contents of newsfeeds but also the subsequent social interactions (and their ripple effects) need to be carefully analyzed and controlled, as the treatment may unexpectedly affect other patients in the OSN. To decide whether a newsfeed is addictive and harmful to SNA patients, a newsfeed selection scheme is required to examine multiple correlated SNA users jointly in order to substitute addictive contents with appropriate ones to incrementally alleviate the SNA symptoms (e.g., to avoid total abstinence). In addition to content filtering, we foster information sharing that leads to benign envy [4], i.e., positive newsfeeds from close friends with different backgrounds may stimulate a positive attitude. By guiding the information flow, severe patients not only find social support easily but also learn how to shape their cognition and alter pathological behaviors.

Complementary to traditional intervention approaches exercised by psychiatrists, alleviating SNAs with the help of data scientists is a new and promising approach for the following reasons. 1) Direct observation. Clinical psychologists usually infer SNA symptoms by indirectly inquiring the patients in clinics. In contrast, user behaviors can be directly observed by analyzing OSN data. 2) Direct treatment. Proper OSN contents can be selected for SNA patients of varied severity to gradually reduce their addictions. ${ }^{6} \mathrm{By}$ contrast, psychiatrists are not trained to select proper OSN contents efficiently and effectively. 3) Joint treatment. For addictions, mental healthcare professionals usually meet one patient at a time to provide the personalized treatment. For effective treatment of SNAs,

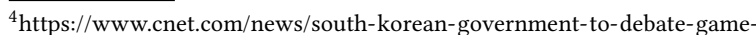
addiction-law

${ }^{5} \mathrm{http}: / / \mathrm{www}$. dailymail.co.uk/news/article-2477401/

${ }^{6}$ While no OSN API is currently available, we demonstrate the technical feasibility later with the browser plugins we developed for this research.
}

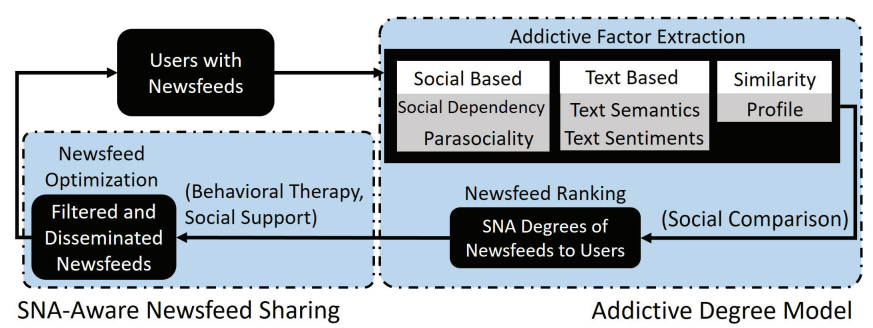

Figure 1: The proposed N3S framework.

in contrast, it is necessary to observe the addictive interactions among users and their friends to stop the reciprocation online. In addition, supportive interactions can be encouraged to alleviate the loneliness, one of the SNA causes.

However, new challenges arise in realizing the data mining approach to alleviate SNAs. First, there exists no special-designed computing system that precisely identifies SNAs and treats users accordingly. Currently, mental healthcare professionals rely mainly on observations of some symptoms, e.g., salience, tolerance, withdrawal, and relapse, to detect SNA cases, while the treatment requires offline counseling. Second, it's challenging to quantitatively measure the addictive degree of a newsfeed to a user because SNAs depend on both the content of the newsfeed and the mental state of the user, as well as the subsequent social interactions with/from other users. Traditional preference-based recommendations cannot quantify the addictive degrees because addictions are different from preferences. For example, one reason of Information Overload in SNAs is compulsive tracing of newsfeeds (though not favorite) from competitors due to malicious envy (e.g., fear to lose) [4]. Third, it is challenging to jointly treat a group of correlated SNA users due to potential impacts on different users and the trade-off in content filtering and feedback support. According to social support theory [27], feedbacks from friends (e.g., likes and comments) on a newsfeed are crucial to remedy loneliness (and addictive behaviors) of the source user (i.e., the author of the newsfeed). Therefore, always filtering the addictive newsfeeds may be beneficial to the browsers but also reduce the chances to help the authors. It is unique in SNAs compared with other addictions.

For the first challenge, we collaborate with domain experts to design a two-phase mining framework, called Newsfeed Substituting and Supporting System (N3S), as shown in Figure 1. N3S, with consents from users, first crawls the newsfeeds users may browse, e.g., recent newsfeeds of friends. To implement online interventions, it is necessary to 1) know the addictive degrees of newsfeeds for each user, and 2) adjust newsfeeds displayed to users in accordance with behavioral therapy, corresponding to the second and third challenges discussed earlier. Accordingly, N3S provides a learning model that quantifies the addictive degrees of newsfeeds to different SNA users and a newsfeed optimization that selects/disseminates the newsfeeds to gradually reduce the addictive contents. For the second challenge, we propose a ranking model called Addictive Degree Model ( $A D M)$ for N3S to quantitatively measure the addictive degrees of newsfeeds to SNA users by extracting unique psychological factors from both the OSN contents and social interactions among OSN users as features. The model is novel and well founded by incorporating the regularization term in accordance with social comparison theory [4] to the ranking objective function, which takes 
into account the negative effect of malicious envy and the positive effect of benign envy on SNAs. For the third challenge, we formulate the SNA-Aware Newsfeed Sharing Problem (SANSP) to address the need of jointly selecting proper newsfeeds for multiple users. The goal is to gradually minimize the maximum total addictive degree of a user with behavioral therapy [29] and encourage the feedbacks with social support theory [27]. We prove that SANSP is NP-Hard and design an algorithm called Randomized Newsfeed Optimization for SANSP (RNOS) with a guaranteed performance bound. Our approach in $R N O S$ optimally allocates the computing budgets (i.e., search of candidate solutions for newsfeed assignments) to different SNA users. Based on practice of behavioral therapy, RNOS properly chooses a set of newsfeeds with gradually lowered addictive degrees to avoid sudden content changes leading to relapse later. RNOS encourages the interactions and feedbacks from friends based on social support theory, and carefully examining trade-offs. The contributions of this paper are summarized as follows.

- We argue that by analyzing and processing OSN data, data scientists have a niche in assisting mental healthcare professionals to alleviate SNAs, thanks to the direct observation, direct treatment, and joint treatment. The proposed N3S is the first data mining framework that leverages behavioral therapy, social comparison theory, and social support theory to alleviate SNAs.

- We point out that addictive degrees cannot be directly quantified by preference-based models, and social interactions are crucial in the addiction and treatment. Therefore, we derive Addictive Degree Model to quantify the addictive degrees of newsfeeds. Moreover, we formulate SANSP and prove that it is NP-Hard. We design a randomized algorithm RNOS with a performance bound to solve SANSP efficiently.

- An extensive user study with 716 Facebook users and 11 mental health professionals manifests that N3S can effectively lower the addictive degree by more than $30 \%$ for severe SNA users. Experiments manifest that the correlation between the SNA scores and the addictive degrees quantified by $\mathrm{ADM}$ is much greater than that quantified by state-ofthe-art preference based models. Moreover, the proposed RNOS outperforms baselines in terms of execution time and objective values on large-scale datasets.

\section{ADDICTIVE DEGREE MODEL}

Addictions, different from preferences, need to emphasize psychological features and mental factors. For example, some SNA users crave for the newsfeeds (even including those not interesting to them) due to loneliness. However, loneliness is not a factor considered in the conventional preference models. To capture the addiction and its degree of severity, in N3S, we consider a number of unique Addictive Features (AFs) according to the mental factors in Psychology, e.g., loneliness and disclosure. Moreover, we add a regularization term to the objective function of addictive degree ranking in accordance with social comparison theory. ${ }^{7}$

\footnotetext{
${ }^{7}$ In Section 4.3, we evaluate ADM with the ground truth via a Facebook user study.
}

\subsection{Addictive Features}

It is important to understand how human mental states are affected by reading different newsfeeds to identify key factors in SNAs. Therefore, we first introduce several simple but unique features in ADM based on Psychology.

2.1.1 Social-based Features. Social dependency. Psychological research manifests that users who obsessively interact with a few OSN friends with strong dependency due to low self-esteem usually suffer from Cyber-Relationship Addiction [6]. Therefore, we analyze the distribution of the interactions among SNA users to extract social dependency. Social dependency of a destination to a newsfeed source is defined as the ratio of the observed interactions between the destination and source to all observed interactions of the destination.

Parasocial relationship. Parasociality represents a one-sided relationship, i.e., only one party cares about the other, and the establishment of parasociality resembles the process of watching TV/reading newspapers [33]. Through acquiring recent status about others, users create an illusionary, non-real sense of intimacy with people, which leads to addictions due to loneliness or other negative emotions [5]. For each newsfeed, the parasocial relationship is measured by $\left|a_{\text {out }}\right| /\left|a_{\text {in }}\right|$, where $\left|a_{\text {out }}\right|$ and $\left|a_{\text {in }}\right|$ denote the number of actions taken from and to each destination, respectively.

2.1.2 Text-based Features. Text semantics. In Psychology, the effect of Echo Chamber and Filter Bubble, strongly related to Information Overload on OSNs [22], states that people naturally fall into different groups and prefer sharing similar opinions with friends. The Internet meme effect also indicates that positive stimuli, such as chatting hot topics, usually reinforce reactions and strengthen friendship [36]. Therefore, we exploit word embedding (Word2Vec [16]) to extract the newsfeed semantics and measure the degree of bursts for a word acting as an Internet meme. For each word $\omega$, we calculate the degree of bursts $b_{\omega}$ for the Internet meme effect, where $b_{\omega}=f s_{\omega} / f l_{\omega}$, and $f s_{\omega}$ and $f l_{\omega}$ are the frequencies of a word $\omega$ appearing in short and long periods of time, respectively. The Internet meme effect is enhanced if a word suddenly becomes popular. Also, we take IDF as a weight to eliminate the effect of stop words. Finally, for each newsfeed $\delta=\left\{\omega_{1}, \omega_{2}, \cdots, \omega_{|\delta|}\right\}$ with $\omega_{i}$ as the $i$-th word in $\delta$, the semantic is modeled as:

$$
\frac{1}{\sum_{i=1}^{|\delta|} \operatorname{IDF}\left(\omega_{i}\right) \cdot b_{\omega_{i}}} \sum_{i=1}^{|\delta|} \operatorname{IDF}\left(\omega_{i}\right) \cdot b_{\omega_{i}} \cdot w 2 v\left(\omega_{i}\right),
$$

where $w 2 v\left(\omega_{i}\right)$ is the Word2Vec embedding, and $\operatorname{IDF}\left(\omega_{i}\right)$ is the inverse-document frequency acquired from the corpus. The weighted average can foster the semantic representation of a passage of texts according to the latent space of word embeddings. Compared with traditional text semantic extraction, ADM emphasizes the meme effect by incorporating $b_{\omega}$ into the word vector. Text sentiment. Recent research indicates that emotion regulations are essential for mental health, and negative emotions are closely related to behavioral addictions [5]. Therefore, we extract text sentiment as a proxy feature. Due to the lack of addictive sentiment labels on newsfeeds, we leverage the unsupervised lexiconbased method to identify the positive and negative polarities of newsfeeds. Based on the seed sentimental polarity [20,25], the 
sentiment scores are propagated to other words according to the similarity in the word embedding space. Moreover, the newsfeed length also acts as a feature since a research on Facebook [38] manifests that long newsfeeds attract more likes and comments. Replies on newsfeeds play crucial roles since the users whose nucleus accumbens in the brain respond more strongly to positive feedbacks are inclined to become addictive [28]. As such, the sentiments of the replies are also extracted as a feature.

\subsection{Derivation of ADM}

After extracting AFs, the next goal is to estimate $d_{u, i}$, the addictive degree of a newsfeed $i$ for user $u$. Specifically, let $\vec{h}_{u, i}$ denote the vector of the extracted AFs for each $u$ and $i$. The existing preferencebased models usually assign $d_{u, i}=\vec{\alpha}_{u} \cdot \vec{\beta}_{i}$, where $\vec{\alpha}_{u}$ and $\vec{\beta}_{i}$ denote the user and item factors derived from $\vec{h}_{u, i}$ [15]. However, AFs suffer from high dimensionality and require dimension reduction to extract latent features. Therefore, motivated by [19], an improved approach is to use a projection matrix $E$ to replace $\vec{\beta}_{i}$ with $E \cdot \vec{h}_{u, i}$, i.e., $d_{u, i}=\vec{\alpha}_{u} \cdot\left(E \cdot \vec{h}_{u, i}\right)$, because the projection matrix $E$ is able to project $\vec{h}_{u, i}$ to a low-dimensional space (feature extraction) and thereby reduces the dimensionality of $\vec{\alpha}_{u}$.

Nevertheless, it is difficult to derive the groundtruth of $d_{u, i}$ for learning since 1) there is no existing questionnaire in Psychology to evaluate the addictive degree of each newsfeed, and 2) addictions to different newsfeeds are internal mental states not explicitly expressed by users. Therefore, we adopt an indirect way to infer the addictive newsfeeds. According to [12], the burst online activity, such as frequent "like" and comments in a short period, is a proxy to SNAs. Therefore, we first detect abnormal burst periods in users' browsing history and then exploit Bayesian Personalized Ranking (BPR) [15] for the optimization of $d_{u, i}$, where $d_{u, i}$ is inclined to be greater than $d_{u, j}$ if user $u$ interacts with newsfeed $i$ in a burst period but she 1) does not interact with newsfeed $j$ or 2) she interacts with newsfeed $j$ in a non-burst period. Through this strategy, we avoid fitting addictive degree to a zero value and increase the volume of effective training data by comparing the newsfeeds. Specifically, given dataset $D=\left\{(u, i, j) \mid u \in U, i \in I_{u}^{+}, j \in I-I_{u}^{+}\right\}$with $U$ and $I$ as the user and newsfeed sets, respectively, and $I_{u}^{+}$is the newsfeed set with the additive candidate activity from $u$, we maximize the probability that $d_{u, i}>d_{u, j}$ by regarding the pairwise error $d_{u, i, j}=d_{u, i}-d_{u, j}$ through the following optimization criterion:

$$
\underset{\theta}{\operatorname{argmin}} \sum_{(u, i, j) \in D}-\ln \left(\sigma\left(d_{u, i, j}\right)\right)+\frac{\gamma_{\theta}}{2}\|\theta\|^{2},
$$

where $\theta$ denotes the set of model parameters $E$ and $\overrightarrow{\alpha_{u}}, \gamma_{\theta}$ is the regularization parameter, $\sigma(\cdot)$ is the sigmoid function mapping $d_{u, i, j}$ from 0 to 1 , and $\ln (\cdot)$ represents the $\log$-likelihood.

To incorporate deeper observations from Psychology for addictions, we propose Addictive Degree Model (ADM) as follows.

$$
\underset{\theta}{\operatorname{argmin}} \sum_{(u, i, j) \in D}-\ln \left(\sigma\left(d_{u, i, j}\right)\right)+\frac{\gamma_{\theta}}{2}\|\theta\|^{2}-\lambda_{s c} \sum_{u \in U, i \in I} \xi_{u, i} \cdot d_{u, i}
$$

where $\xi_{u, i}$ denotes the regularization factor for a newsfeed $i$ to user $u$ according to social comparison theory, and parameter $\lambda_{s c}$ controls the impact of social comparison. Specially, social comparison plays a crucial role here because Psychology research manifests that users exposed to positive posts usually feel malicious envy or benign envy due to social comparison [4]. Many people are used to evaluate and compare their achievements with those of friends, especially now status updates are very convenient on OSNs. Malicious envy is triggered by comparisons among friends with similar backgrounds and usually incurs Information Overload and Net Compulsion, because a person in this case tends to feel pressured and then frequently checks the updates of friends. In contrast, benign envy for close friends with different backgrounds will lead to an elevating motivation.

To model malicious and benign envy, we exploit sentiment analysis to derive the positive polarity Pos $_{i}$ for each newsfeed $i$ and then calculate the profile similarity and social familiarity between friends. Let $v_{i}$ denote the author of newsfeed $i$. Each user $u$ is associated with a profile vector $\vec{\phi}_{u}$, and the similarity score between users $u$ and $v, \operatorname{sim}\left(\vec{\phi}_{u}, \vec{\phi}_{v}\right)$, ranges in [-1,1]. Also, each user pair $(u, v)$ is associated with a familiarity score $\tau_{u, v}$. Let $\xi_{u, i}$ set as $\operatorname{Pos}_{i} \cdot \tau_{u, v_{i}} \cdot \operatorname{sim}\left(\vec{\phi}_{u}, \vec{\phi}_{v_{i}}\right)$ in the regularization term of Eq. (3), such that malicious envy appears from a positive newsfeed $i$ (i.e., $\operatorname{Pos}_{i}>0$ ) for user $u$ when $u$ and $v_{i}$ are familiar (i.e., large $\tau_{u, v_{i}}$ ) and with similar profiles [4]. However, this term will not increase the addictive degrees of all newsfeeds because the first two terms will also increase. On the other hand, benign envy appears when $u$ and $v_{i}$ have different profiles, i.e., similarity is negative. Since the third term becomes positive, decreasing $d_{u, i}$ reduces the objective value, and thus the probability of selecting newsfeed $i$ to encourage user $u$ increases afterward. Therefore, the above model is able to incorporate the characteristics of SNAs and alleviate the data sparsity problem, because the users with fewer activities can benefit from the auxiliary information of social comparison.

By minimizing Eq. (3), the likelihood of the observed ranking can be maximized, and we exploit the stochastic gradient descent algorithm to effectively learn the model parameters. Specifically, the first derivative of $\ln \left(\sigma\left(d_{u, i, j}\right)\right)$ w.r.t. $\theta$ is

$$
\begin{aligned}
& \frac{\partial \ln \left(\sigma\left(d_{u, i, j}\right)\right)}{\partial \theta}=\frac{\partial \ln \left(\sigma\left(d_{u, i, j}\right)\right)}{\partial \sigma\left(d_{u, i, j}\right)} \frac{\partial \sigma\left(d_{u, i, j}\right)}{\partial d_{u, i, j}} \frac{\partial d_{u, i, j}}{\partial \theta} \\
= & \frac{1}{\sigma\left(d_{u, i, j}\right)} \sigma\left(d_{u, i, j}\right)\left(1-\sigma\left(d_{u, i, j}\right)\right) \frac{\partial d_{u, i, j}}{\partial \theta}=\sigma\left(-d_{u, i, j}\right) \frac{\partial d_{u, i, j}}{\partial \theta} .
\end{aligned}
$$

Therefore, $\theta$ is updated by

$$
\theta \leftarrow \theta-l r \cdot\left(\sigma\left(-d_{u, i, j}\right) \frac{\partial d_{u, i, j}}{\partial \theta}+\gamma_{\theta} \theta-\lambda_{s c} \xi_{u, i} \frac{\partial d_{u, i}}{\partial \theta}\right),
$$

where $l r$ is the learning rate. Through the learning process, ADM finds the users' personalized latent factor and appropriate projection matrix on AFs to accurately model the addictive degrees. In Section 4.2, we evaluate the learned addictive degrees with the ground truth in Psychology.

\section{SNA-AWARE NEWSFEED SHARING}

Equipped with ADM, the proposed N3S, based on the practice of behavioral therapy and social support theory in Psychology, explores joint recommendations of suitable newsfeeds for multiple SNA users. As discussed earlier, typical recommendation schemes examine user preferences and thereby focus on the individual or group needs. In contrast, for SNAs, the impacts of each newsfeed to each user are three-fold. For the destination user that reads the 
newsfeed, 1) the preference and 2) the addiction of the user to the newsfeed can be different as explained in Section 2.2. For the source user that shares the newsfeed, 3) the interactions and feedbacks from the destination users reading the newsfeed also play crucial roles as the social supports to alleviate the addictions of the source user. In other words, it is preferred to give a higher priority to the newsfeed with the higher preference but lower addiction. Moreover, it is important to carefully examine and evaluate the addictions not only from the aspect of OSN contents to different SNA users but also the potential triggered feedbacks and interactions among friends. When we select and assign a newsfeed from a source to a destination, it is necessary to carefully examine the trade-off between the increment of SNA for the newsfeed destination (i.e., cost to the destination) after reading it and the decrement of SNA to the newsfeed source (i.e., profit to the source) due to the potential feedbacks as the social supports. The above trade-off is unique and has not been explored by previous research on recommendations.

To effectively address the above issues, we formulate a new research problem, named SNA-Aware Newsfeed Sharing Problem (SANSP), to minimize the maximum total addictive degree of any user without compromising the user preferences, while the ideas of tailoring treatment based on behavioral therapy [29] and social support theory [27] are incorporated in SANSP. We prove that SANSP is NP-Hard. To solve $S A N S P$, we propose a randomized algorithm, RNOS (Randomized Newsfeed Optimization for SANSP), with a guaranteed performance bound. It exploits Optimal Computing Budget Allocation (OCBA) [11], which is an efficient and effective approach to solve challenging NP-Hard problems. For each user, it randomly generates a few initial partial solutions (a set of newsfeeds with the size smaller than $p$ ) and iteratively selects a newsfeed according to the probability derived from Importance Sampling [34], to evaluate the corresponding total addictive degrees. To avoid the sudden changes of the assigned newsfeeds, which may lead to anxiety according to behavioral therapy, RNOS selects the newsfeed sets that gradually reduces the total addictive degrees as implemented in behavioral therapy for newsfeed destination users, whereas the interactions and feedbacks from friends are encouraged for newsfeed source users based on social support theory. To minimize the maximum total addictive degree of any user, the patients with severe addictions are treated with priorities to reduce their total addictive degrees, by carefully selecting the newsfeeds shared to them and encouraging the social support for them. To avoid trapping in locally optimal solutions, RNOS optimally invests more computing resources to the users with potentially higher addictive degrees to help them search a better newsfeed assignment. The difference of the preference and addiction, as well as the trade-off between addiction (to the newsfeed destination) and social support (to the newsfeed source), are carefully examined by RNOS to properly assign the newsfeeds to all users.

\subsection{SANSP Problem}

Given a social network $G=(V, E)$ of $n$ users, where each node $v_{u} \in V$ denotes a user $u$, the decision tensor is $X$, where each element $x_{u, v, w} \in X$ is a binary decision variable dictating whether the $w$-th newsfeed from user $v$ is recommended to user $u$, and $a_{u, v, w}$ is the corresponding addictive degree derived from Section
2. We first formulate a basic form of SANSP as follows.

$$
\begin{gathered}
\min _{1 \leq u \leq n}\left\{f_{u}(X)\right\} \\
\text { s.t. } \quad \sum_{v} \sum_{w} x_{u, v, w}=p, \\
\sum_{v} \sum_{w} g_{u}\left(x_{u, v, w}\right) \geq \Omega_{u}, \forall u,
\end{gathered}
$$

where $f_{u}(X)=\sum_{v} \sum_{w} x_{u, v, w} a_{u, v, w}$ is the total addictive degree of user $u$, and $p$ is the number of newsfeeds displayed to each user, i.e., the top- $p$ newsfeed recommendation; ${ }^{8} g_{u}(X)$ is the preference of user $u$ to different newsfeeds, which can be derived from preference-based models; and $\Omega_{u}$ is the threshold to be lowered gradually in accordance with behavioral therapy [29] in order to avoid sudden change of contents that may discourage the user from continuing the SNA treatment. ${ }^{9}$ An alternative objective is to minimize the total addictive degrees of all users, which however tends to induce the imbalanced addiction distribution among users. Notice that the proposed objective function, i.e., minimizing SNAs of the most severe patient with the helps from other users, has been widely adopted in related therapies [30]. ${ }^{10}$ Clinicians usually request patients to form a healthier peer network to help the patients with severe symptoms learn positive behaviors from peers.

In the above problem formulation, however, social support theory is not considered in the total addictive degree $f_{u}(X)$. Psychology research shows that the recovery-oriented social support fosters greater self-efficacy toward ongoing abstinence because recovering persons can acquire effective coping strategies from their peers [27]. Moreover, users that have not developed a healthy relationship network are more inclined to relapse according to the famous Rat Park experiment [9]. Therefore, we add a regularization term to the objective function as follows.

$$
\sum_{v} \sum_{w} x_{u, v, w} a_{u, v, w}-\lambda_{1} \sum_{v} \sum_{w} \tau_{u, v} S U P\left(x_{v, u, w}\right),
$$

where $\lambda_{1}$ controls the extent of social support, and $\operatorname{SUP}\left(x_{v, u, w}\right)$ denotes the expected support that user $u$ receives by sending her $w$-th newsfeed to user $v .{ }^{11}$ SANSP is unique and challenging (NPHard) due to the trade-off between social support and addictive degrees. That is, always filtering the addictive newsfeeds may be beneficial to the destination user but also reduce the potential social support to help the source user.

\section{THEOREM 1. SANSP is NP-Hard.}

Proof. We prove the theorem with the reduction from Partition Problem (PP) [24], which decides whether a given multiset $S$ of positive integers can be divided into two subsets $S_{1}$ and $S_{2}$ such that the total weight of $S_{1}$ equals to that of $S_{2}$. For each instance of $P P$, we construct an instance for $S A N S P$ with $G=(V, E)$ including three nodes $u, v$, and $w$ and two directed edges from $u$ to $v$ ( $v$ follows $u$ ) and from $w$ and $u$ (u follows $w$ ), respectively, where $p=|S|$. Moreover, user $u$ has $2|S|$ newsfeeds with $a_{v, u, k}=\operatorname{SUP}\left(x_{v, u, k}\right)=s_{k}$ for $k=1, \cdots,|S|$, where $s_{k}$ is the $k$-th element in $S$. For $k=|S|+1, \cdots, 2|S|$, the support and addictive

\footnotetext{
${ }^{8}$ It is possible to assign a different $p$ to each user according to her browsing behavior. ${ }^{9}$ It is also possible to revise the constraint and $\Omega_{u}$ to represent the total addictive degree (instead of the preference) to avoid a sudden content change.

${ }^{10}$ SNAs of other users are also reduced to avoid becoming the most severe patient.

${ }^{11} S U P\left(x_{v, u, w}\right)$ can be estimated by analyzing historical data, i.e., the probability that user $v$ responds to user $u$ 's newsfeeds.
} 
degrees are zero. Therefore, when we select any newsfeed from $u$ to $v$, the addictive degree increased to $v$ is identical to the addictive degree reduced from $u$ (due to the social support from $v$ ), where $\lambda_{1}=\tau_{i, j}=1$. Moreover, user $w$ has $|S|$ newsfeeds with the total addictive degree $\sum_{k=1}^{|S|} a_{u, w, k}=S U P\left(x_{u, w, k}\right)=\sum_{k} s_{k}$. Finally, the threshold of user preferences is $\Omega_{i}=-\sum_{k} s_{k}$ for all users.

We first prove the sufficient condition. For each instance of $P P$ with $S_{1}$ and $S_{2}$ sharing the same total weight $\eta$, we let $x_{v, u, k^{\prime}}=1$ for every element $k^{\prime}$ in $S_{1}$. We further select $|S|-\left|S_{1}\right|$ newsfeeds from $k=|S|+1, \cdots, 2|S|$ to satisfy the size constraint. The objective of SANSP is also $\eta$ since the total addictive degrees of $v, u$, and $w$ are $\eta$ (newsfeeds from $u$ ), $2 \eta$ (newsfeeds from $w$ ) $-\eta$ (social support from $v$ ), and $-\eta$ (social support from $u$ ), respectively. In contrast, if directly selecting the $|S|$ newsfeeds from $k=|S|+1, \cdots, 2|S|$ will create an inferior solution with the total addictive degree of $v, u$, and $w$ as $0,2 \eta$, and $-\eta$, respectively, so that the maximum one is $2 \eta$.

We then prove the necessary condition. For each instance of $S A N S P$, if the objective is $\eta$, the total addictive degree of $v$ cannot be smaller than $\eta$; otherwise, that of $u$ will exceed $\eta$. $S_{1}$ can be constructed by the selected newsfeeds for $v$, and $S_{1}$ and $S_{2}$ share the same total weight. The theorem follows.

\subsection{Algorithm Design for SANSP}

One greedy approach for SANSP is to select the newsfeed incurring the smallest increment of the total addictive degree at each iteration to each user $u .^{12}$ However, it does not consider the benefits of feedbacks in social support theory. Also, greedy algorithms for an NP-Complete problem tend to be trapped in a local optimum To address the above issues, we design a randomized algorithm, RNOS (Randomized Newsfeed Optimization for SANSP), to select multiple newsfeeds with different probabilities for each user to expand each partial solution (a set of newsfeeds with a size less than p) toward multiple directions. The probabilities can be optimally derived according to previous sampling results.

To effectively allocate the numbers of samples for partial solutions, RNOS exploits the framework of Optimal Computing Budget Allocation (OCBA) [11] to invest more computing resources (i.e., more samples) to users with larger potentials to minimize the objective eventually. In other words, it allocates more computing budgets (i.e., generate more candidate solutions) for treating severe patients, but the preferences and addictive degrees of others are adequately handled. RNOS exploits OCBA to search the best trade-off and jointly treat multiple patients.

The trade-off between the solution quality and execution time in $R N O S$ is controlled by $T$, which is the number of randomly generated solutions. ${ }^{13}$ Later we prove that RNOS optimally divides $T$ into $n$ parts for the $n$ users and provides a performance guarantee in Theorem 3. Specifically, RNOS includes two steps. (1) Initial Sampling. For each user, it first randomly generates a few final solutions as samples by iteratively selecting a random newsfeed and adding it to a partial solution. (2) Budget Allocation. It evaluates the addictive degree of each user in the above final solutions and derives the computing resources optimally allocated to each user. Let $J_{u}$ and $J_{u}^{*}$ denote the random variables of the addictive degree sampled

\footnotetext{
${ }^{12}$ The above algorithm is called DGreedy in Section 4.

${ }^{13}$ The setting of $T$ is studied in Section 4.
}

from a final solution expanded from node $v_{u}$ and the minimal addictive degree expanded from node $v_{u}$, respectively. To optimally allocate the computing budgets for each user, let the solution quality $Q$ denote the maximum of the minimal addictive degrees sampled from all users, i.e., $Q=\max \left\{J_{1}^{*}, J_{2}^{*}, \ldots, J_{u}^{*}, \ldots, J_{n}^{*}\right\}$. Since $J_{u}^{*}$ of node $v_{u}$ is related to the number of generated final solutions $N_{u}$, the computing budget allocation problem is as follows.

$$
\begin{gathered}
\min _{N_{1}, N_{2}, \ldots, N_{n}} Q, \\
\text { s.t. } N_{1}+N_{2}+\ldots+N_{n}=T .
\end{gathered}
$$

Previous research on OCBA [11] manifests that the distribution of $J_{u}$ is similar to the Gaussian distribution. ${ }^{14}$ Therefore, the probability density function (PDF) and cumulative distribution function (CDF) for $J_{u}$ are as follows.

$$
\begin{aligned}
& p_{J_{u}}(x)=\phi\left(\frac{x-\mu_{u}}{\sigma_{u}}\right)=\frac{1}{\sigma_{u} \sqrt{2 \pi}} e^{-\frac{1}{2}\left(\frac{x-\mu_{u}}{\sigma_{u}}\right)^{2}} . \\
& P_{J_{u}}(x)=\Phi\left(\frac{x-\mu_{u}}{\sigma_{u}}\right)=\frac{1}{2}\left(1+\operatorname{erf}\left(\frac{x-\mu_{u}}{\sigma_{u} \sqrt{2}}\right)\right) .
\end{aligned}
$$

Since the CDF of $J_{u}^{*}$ is $P_{J_{u}^{*}}(x)=1-\left[1-P_{J_{u}}(x)\right]^{N_{u}}$, its PDF is $p_{J_{u}^{*}}(x)=N_{u} P_{J_{u}}(x)^{N_{u}-1} p_{J_{u}}(x)$. Let $v_{b}$ be the bottleneck node that generates the maximum objective value. The probability that $J_{u}^{*}$ is greater than $J_{b}^{*}$ is given as follows. ${ }^{15}$

$$
\begin{gathered}
p\left(J_{b}^{*}-J_{u}^{*} \leq 0\right)=1-\int_{-\infty}^{\infty} p_{J_{b}^{*}}(x) P_{J_{u}^{*}} d x \\
=1-\int_{-\infty}^{\infty} N_{b} P_{J_{b}}(x)^{N_{b}-1} p_{J_{b}}(x)\left\{1-\left[1-P_{J_{u}}(x)\right]^{N_{u}}\right\} d x .
\end{gathered}
$$

The second phase allocates the computing budget to user $u$ and $b$ according to the ratio of $p\left(J_{b}^{*} \leq J_{u}^{*}\right)$. More specifically, this phase is divided into $r$ stages. In each stage, since the real values of $v_{b}$, $\mu_{u}$ and $\sigma_{u}$ are unknown during sampling, we first approximate it according to the sampled results in the previous stages. Intuitively, when the total addictive degree of newsfeeds displayed to user $u$ is large in current stage, user $u$ will be allocated with more budgets (generate more candidate newsfeed sets) to find a better solution. However, lowering the total addictive degree of $u$ may reduce the social support from the neighboring users. Moreover, when user $v$ is not addictive but neighboring to many severe SNA users, assigning more newsfeeds to $v$ from neighbor nodes may effectively alleviate the SNAs of neighbors. Therefore, it is necessary for RNOS to carefully assign the newsfeeds with different probabilities.

After allocating the computing budget to each user, RNOS exploits the cross-entropy method [34] based on Importance Sampling to adaptively assign a different probability to each newsfeed for each user from the sampled results in previous stages. Let $\vec{p}_{u, t}$ denote the newsfeed selection probability vector for user $u$ in stage $t$, i.e., $\vec{p}_{u, t}=\left\langle p_{u, 1,1, t}, p_{u, 1,2, t}, \ldots, p_{u, v, w, t}, \ldots, p_{u, n, k_{n}, t}\right\rangle$, where $p_{u, v, w, t}$ is the probability of choosing the $w$-th newsfeed of user $v$ for user $u$ in stage $t$, and $k_{n}$ is the number of newsfeeds for user $n$. In the first stage, $\vec{p}_{u, 1}$ for each user $u$ is initialized uniformly for every newsfeed, i.e. $\vec{p}_{u, v, w, 1}=p / \sum_{v} k_{v}, \forall v_{v} \in G, v_{v} \neq v_{u}$, where $p$ is the size constraint. Let Bernoulli sample vector $\vec{X}_{u}^{(q)}$

\footnotetext{
${ }^{14}$ Our user study also indicates that the distribution of $J_{u}$ is similar to the normal distribution, which is presented in [1] due to the space constraint.

${ }^{15} p\left(J_{b}^{*} \leq J_{u}^{*} \leq 0\right)$ will be used to derive the budget allocation. With more computing budget, the probability of finding the real $v_{b}$ approaches 1 .
} 
$=\left\langle x_{u, 1,1}^{(q)}, \ldots, x_{u, v, w}^{(q)}, \ldots, x_{u, n, k_{n}}^{(q)}\right\rangle$ denote the $q$-th sample vector for user $u$, where $x_{u, v, w}^{(q)}$ is 1 if the $w$-th newsfeed of user $v$ for $u$ is selected in the $q$-th sample, and 0 otherwise. RNOS calculates the objective value $S\left(\vec{X}_{u}^{(q)}\right)$ of all newsfeeds assigned to user $u$ in the $q$-th sample. For each user $u$, it then sorts $S\left(\vec{X}_{u}^{(q)}\right)$ in an ascending order. Let $\gamma_{u, 1}$ denote the objective value of the top- $\rho$ performance sample. The selection probability $p_{u, v, w, t+1}$ of the $w$-th newsfeed of user $v$ in the next stage is derived as follows,

$$
p_{u, v, w, t+1}=\frac{\sum_{q=1}^{N_{u, t}} I_{\left\{S\left(\vec{X}_{u}^{(q)}\right) \leq \gamma u, t\right.} x_{u, v, w}^{(q)}}{\left.\sum_{q=1}^{N_{u, r}} I_{\left\{S\left(\vec{X}_{u}^{(q)}\right) \leq \gamma u, t\right.}\right\}},
$$

where $N_{u, t}$ denotes the total number of samples for user $u$ in the $t$-th stage, and the indicator $I_{\left\{S\left(\vec{X}_{u}^{(q)}\right) \leq \gamma_{u, t}\right\}}$ is 1 if the objective value of $\vec{X}_{u}^{(q)}$ is smaller than $\gamma_{u, t}$, and 0 otherwise. Intuitively, $p_{u, v, w, t+1}$ is the proportion of times that the $w$-th newsfeed of $v$ is sent to $u$ in the samples with small objective values $\left(\leq \gamma_{u}, t\right)$. Eq. (4) effectively minimizes the Kullback-Leibler cross entropy (KL) distance [34] between the newsfeed selection probability $\vec{p}_{u, t}$ and the distribution of top- $\rho$ performance samples, such that the solutions in stage $(t+1)$ is guaranteed to be the closest to the top- $\rho$ performance samples in stage $t$. Therefore, by picking the top- $\rho$ performance samples to generate the solutions in the next stage, the performance can be improved iteratively. In the following, we first examine the role of $v_{b}$ and then derive the performance bound. We prove that the probability assignment scheme is optimal from the perspective of cross entropy in [1] with a similar way as [34].

Theorem 2. For SANSP with parameter $(n, T)$, where $n$ is the number of users and $T$ is the total computing budget, the probability $P_{b}$ that $v_{b}$ is actually the bottleneck user (generating the highest objective value) is at least $1-\frac{1}{n-1} \sum_{k=0}^{N_{u}}(-1)^{k} C_{k}^{N_{u}} \frac{N_{b}}{k+N_{b}}$.

Proof. The proof is shown in [1] due to the space constraint.

TheOREM 3. For SANSP with $r$ stages, the expected maximum objective value $E[Q]$ of RNOS is at most $\mu_{b}-\sigma_{b} \Phi^{-1}\left(\frac{N_{b}-\zeta}{N_{b}-2 \zeta+1}\right)+$ $\sum_{u=1}^{n} \sigma_{u}$, where $N_{b}$ after $r$ stages is $\frac{4+n(r-1)}{4 r n} T$.

Proof. The proof is shown in [1] due to the space constraint.

\section{EXPERIMENTAL RESULTS}

In this section, we evaluate $A D M$ and $R N O S$ with three real datasets and carry out a user study. ${ }^{16}$ Notice that the proposed N3S is designed to be generally applicable to OSNs similar to Facebook.

\subsection{Data Preparation and Evaluation Plan}

We recruit 716 Facebook users around the world via Amazon MTurk (483) and RapidWorkers (233) and 11 mental health professionals, including 5 psychiatrists and 6 clinical psychologists from California School of Professional Psychology, California Institute of

\footnotetext{
${ }^{16}$ The IRB number of this project is AS-IRB-HS07-106009. The three real datasets and the user study data, after being processed to satisfy the IRB privacy requirements, can be downloaded at [1].
}

Integral Studies, City College of New York, Washington University in St. Louis, King's College in London, Taipei City Hospital, National Taipei University, and psychiatric clinics. Participants of the user study include 409 males and 307 females from universities, government offices, technology companies, art centers, banks, and businesses. Each user installs a Facebook crawler we implement for collecting newsfeeds and a Google Chrome plugin for displaying the selected newsfeeds and recording the browsing behaviors of the participants in our study. ${ }^{17}$ Users periodically take standard questionnaires [10] in Self Report Inventory (SRI) for evaluation by mental healthcare professionals to label the SNA scores, i.e., ground truth. ${ }^{18}$ To evaluate ADM, we compare ADM (with different weights $\lambda_{s c}$ in social comparison), GBPR [32], and RLFM [2] (two preference-based latent factor models). Since there is no existing questionnaire in Psychology to quantify the addictive degree of each newsfeed, we analyze the correlation between the total addictive degrees (derived from models) and the SNA scores (labelled by SRI questionnaires and mental healthcare professionals) with two correlation metrics. ${ }^{19}$

In the evaluation of N3S, users are categorized by the questionnaire (adjusted by mental health professionals) as 1) none or mild (average users with normal or slightly over usage but still under control), 2) moderate (users experiencing occasional or frequent problems), or 3) severe (users experiencing serious problems in their life). We compare the following approaches: 1) newsfeed substituting and supporting system (N3S), 2) greedy approach (DGreedy) mentioned in Section 4.2,3) aversive approach (AVS) that sends the top- $p$ newsfeeds with the least addictive degrees, 4) abstinence approach (ABS) that prohibits SNA users from accessing OSNs during 5 weekdays but allowing them during the weekends, and 5) normal approach (NORM) that sends the original newsfeeds in Facebook. DGreedy first sorts the newsfeeds in a descending order of $g_{u}$ (i.e., preference of user $u$ ) and iteratively selects the newsfeeds until the total preference meets the preference threshold $\Omega$ or the number constraint $p$. If the number constraint $p$ is not satisfied, it then selects the newsfeeds in an ascending order of addictive degrees until $p$ newsfeeds are extracted. ${ }^{20}$ In contrast, AVS iteratively selects the newsfeeds in an ascending order of addictive degrees, but it may not always satisfy the preference constraint. The numbers of users assigned to N3S, DGreedy, AVS, ABS, and NORM are 145, 143, 142, 143, and 143, respectively. By default, the average number of newsfeeds $p$ is 40 , and the threshold $\Omega$ is $75 \%$ of the previous threshold in every 7 days according to behavioral therapy [29]. We collect the average online frequency per day, average duration per day, and the SNA scores ${ }^{21}$ summarized from the standard questionnaires.

\footnotetext{
${ }^{17}$ Mobile Facebook accesses are not allowed because Android does not support Chrome plugins. We detect prohibited mobile accesses by checking if the Chrome access log is the same as the complete browsing history obtained from Facebook APIs. Once detected, mental healthcare professionals lower $\Omega_{u}$ in the behavioral therapy.

${ }^{18}$ Researchers [10] recommend a polythetic cut-off approach (scoring 3 or more on at least four of six items are potential SNAs). The validity of SRI here shows strong internal consistency $(\alpha=.90-.93)$ and good test-retest reliability $(\gamma=0.85)$.

${ }^{19}$ The social support is not considered here because it is the same for different models and does not affect the correlation (constant shift).

${ }^{20}$ DGreedy and N3S can be regarded as an A/B test that evaluates the impact of the social support.

${ }^{21}$ The SNA score measures the overall addiction of each user to Facebook, whereas the addictive degree represents the user's addiction to individual newsfeed.
} 


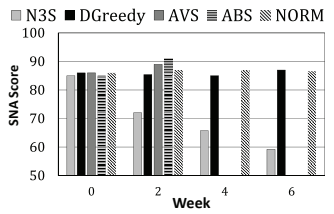

(a) SNA score (severe).

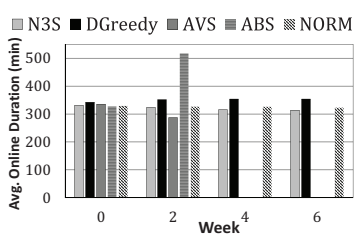

(c) Avg. duration (severe)

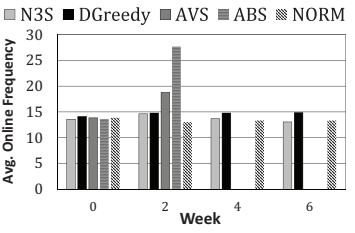

(b) Avg. online freq. (severe).

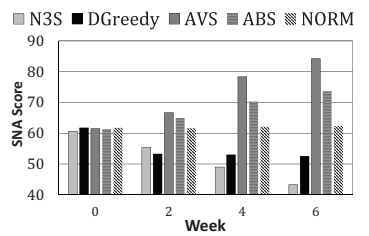

(d) SNA score (moderate)
Figure 2: Comparisons of different severity indices.

For large-scale experiments, three real datasets are included to evaluate the efficacy (objective function) and efficiency (execution time) of different algorithms for SANSP: 1) Twitter [14] with 3M nodes and more than $50 \mathrm{M}$ tweets. Each user on average has 14.8 friends and 14.9 followers. 2) Instagram with $2 \mathrm{~K}$ users, $9 \mathrm{M}$ tags, $1200 \mathrm{M}$ likes, and $41 \mathrm{M}$ comments [18]. 3) Flickr with $1.8 \mathrm{M}$ nodes and $22 \mathrm{M}$ edges. ${ }^{22}$ Since there is no newsfeed in the Instagram and Flickr datasets, the addictive degrees are approximated according to their social-based features. We compare RNOS with DGreedy, randomized greedy (RGreedy) ${ }^{23}$, and GBPR with the top- $p$ preference recommendation [32] (GBPR-TOP-p). The total SNA degree of each user is normalized by the maximum total SNA degree of all users, where $T$ is 100 times of the network size with 5 stages $(r=5)$. The default cross-entropy parameter $\rho$ in RNOS is 0.3 [34]. We also implement a parallel $R N O S$ to evaluate the gain with more CPUs in an HP DL580 server with 4 Intel $2.4 \mathrm{GHz}$ CPUs.

\subsection{User Study}

Quantitative Analysis. Figure 2(a) presents the SNA scores of severe users, where week 0 denotes the time when the experiment starts. As shown, the reduction of SNA scores is more significant for N3S compared with other approaches. All ABS users quit the experiments after two weeks even though we allow them to access ordinary OSNs during the weekends, because highly addictive users usually are not able to delay gratification, i.e., to resist the temptation for an immediate reward [3]. The AVS users also quit the experiments after two weeks because the newsfeeds are much less addictive than before. Therefore, sudden content changes are not suitable for severe users. Figures 2(b) and 2(c) analyze the average online frequency and duration per day, respectively. The online frequencies of $A B S$ users are much greater due to the relapse, i.e., reverting back to the excessive usage of OSNs after abstinence. Note that the duration of N3S users only slightly decreases, manifesting that N3S minimizes the addictive degrees but retains the users' preferences for OSNs. Figure 2(d) presents the SNA scores of

\footnotetext{
${ }^{22}$ http://socialnetworks.mpi-sws.org/data-imc2007.html.

${ }^{23}$ RGreedy improves DGreedy by iteratively replacing a selected newsfeed with an unselected one with the probability proportional to the decrement of the addictive degree.
}

moderate users. Although all AVS users do not quit the experiment, their SNA scores gradually approach the severe level (80) because the newsfeeds with the least addictive degrees do not satisfy the users, i.e., AVS users usually crave for more newsfeeds. In contrast, the SNA scores of the N3S users gradually decrease.

Moreover, all participating mental healthcare professionals are asked two questions in Likert scale: Q1) Is the proposed system helpful for SNAs? Q2) Is the proposed system promising to be a tool for clinical interventions in the future? With detailed explanations of all algorithms and free access to users' social logs and SNA scores, all the mental healthcare participants agree (45\%) or strongly agree $(55 \%)$ that N3S is helpful for SNAs. They also agree $(64 \%)$ or strongly agree $(36 \%)$ for Q2. Especially, the professionals find a severe case that user A, a 24-year-old female living separately with her boyfriend and family, frequently posts newsfeeds to seek social support, such as "What cannot be cured must be endured" with her laptop/mug photos on her Facebook timeline more than 3 times a week. However, these posts usually attract less than 5 likes. With N3S, the SNA score of A decreases by $25.88 \%$. The number of her newsfeeds for seeking social support is down to 1.2 per week at the end of study, while attracting more than 11 likes, which shows that social support is important to alleviate SNAs.

Qualitative Analysis. Benign envy (stimulates a positive attitude) and malicious envy (stimulates a negative attitude due to peer pressure) play important roles in reducing the SNA reduction. For example, the SNA score of user A, 34-year-old male as an academic researcher, is 81 at the beginning of the user study. His answers to the standard questionnaire reveal that he usually spends much more time than he expects and even loses sleep due to late-night access on Facebook to check others' newsfeeds. Through the interview, user A explains that he sometimes just wants to read some newsfeeds before the sleep but eventually feels compulsive to read more and even go to their personal web pages to find whether his colleagues publish new papers or receive awards. We first analyze the social $\log$ of user A and observe that he usually interacts with users with similar backgrounds (92\%). After 6 weeks, the SNA score of user $\mathrm{A}$ is reduced to 62 . We further analyze the historical newsfeeds and have the following observations. 1) ADM quantifies the addictive degrees of the positive newsfeeds from users with similar backgrounds as high values (in top 20\%) and the positive newsfeeds from users with different backgrounds as low values (bottom $10 \%$ $40 \%)$. 2) N3S reduces the positive newsfeeds from users with similar backgrounds (-48\%) and encourages the positive newsfeeds from users with different backgrounds $(+34 \%)$. Through the interview with user A, he recalls that the frequency of losing sleep and checking others' personal websites becomes lower, because N3S reduces the chance of triggering the malicious envy. In contrast, preference models encourage the positive newsfeeds from users with similar backgrounds since user A interacts with those newsfeeds frequently but it may incur addictions due to malicious envy.

On the other hand, social support also plays an important role. For example, in our user study, we find user B, a 27-year-old male, with the SNA score as 85 at the beginning of the user study. We first analyze the social $\log$ of $\mathrm{B}$ and observe that he frequently replies to others' newsfeeds (11.7 per day), but others do not respond to his comments (reply rate is $4.32 \%$ ). Through the interview, B explains that he follows many female Facebook celebrities. After 6 weeks, 
Table 1: Correlation analysis of different approaches.

\begin{tabular}{|l|ll|}
\hline & Pearson & Spearman \\
\hline \hline GBPR & 0.3020 & 0.4235 \\
RLFM & 0.3532 & 0.4295 \\
ADM $\left(\lambda_{S C}=0.01\right.$, Seman- & $0.8458^{*}$ & $0.8386^{*}$ \\
tics+Sentiment+Social) & & \\
\hline ADM $(0.001)$ & 0.8260 & 0.8014 \\
ADM $(0.0001)$ & 0.7549 & 0.7884 \\
ADM $(0)$ & 0.7402 & 0.7485 \\
\hline Semantics & 0.6613 & 0.6110 \\
Semantics+Sentiment & 0.7487 & 0.7343 \\
Semantics+Social & 0.7210 & 0.7363 \\
\hline
\end{tabular}

the SNA score of user B is reduced to 67. We trace the historical newsfeeds and find out that 1) ADM utilizes social dependency to quantify the newsfeeds from users who do not reply to his comments as high values (in top 32\%), and 2) N3S then reduces the newsfeeds from those celebrities $(-78 \%)$ and encourages the newsfeeds from users who are inclined to reply his comments $(+37 \%)$. In this case, N3S successfully increases the chance of social support and reduces the loneliness, which is the primary reason for SNA as reported by B. Therefore, the above study manifests that N3S is able to effectively alleviate SNA with different causes of addictions.

\subsection{Analysis of ADM}

To evaluate the effectiveness of ADM, we first analyze the correlation between the addictive degrees (derived from models) and the SNA scores (labelled by SRI questionnaires and mental healthcare professionals) with two correlation metrics: Pearson's correlation coefficient and Spearman's rank correlation coefficient. Pearson's correlation coefficient measures the linear correlation for two variables, while Spearman's correlation focuses on the rank correlation. Table 1 first compares ADM, GBPR [32], and RLFM [2] (two preference-based latent factor models). The results manifest that the correlation for ADM is high in all metrics, and the differences between ADM and preference-based methods are statistically significant ( $p$-value $<0.05)$, because ADM is designed to capture SNAs by considering AFs and incorporating social comparison theory. We further compare ADM with different $\lambda_{s c}$ in social comparison. $\mathrm{ADM}$ strikes the best balance when $\lambda_{s c}$ is equal to 0.01 . Therefore, $\lambda_{s c}$ is set as the default for the rest of evaluation. Moreover, ADM without social comparison $\left(\lambda_{s c}=0\right)$ still outperforms GBPR and RLFM due to the extracted AFs. Finally, we analyze the feature effectiveness of AFs, which shows that semantics, sentiment, and social-based features all contribute to ADM.

\subsection{Performance Comparison for RNOS}

Figures 3(a)-3(b) compare DGreedy, RGreedy, RNOS and GBPR-TOP-p on large-scale datasets in terms of execution time and objective values. The total addictive degree of a user is normalized with the maximum total addictive degree among all users. Figure 3(a) indicates that RGreedy is computationally expensive since it needs to sum up the addictive degrees of newsfeeds many times during the newsfeed selection process to find the proportions of decrement in addictive degrees. When $p$ is greater than 20, RGreedy still does
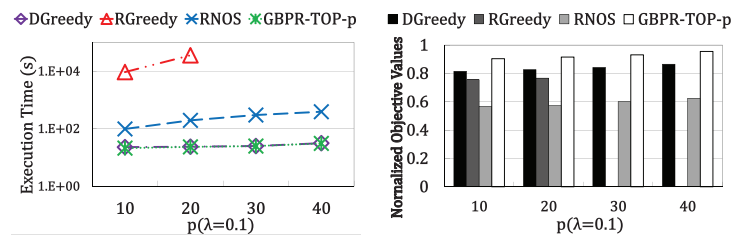

(a) Execution time with diff. p. (Twit- (b) Obj. values with diff. p. (Twitter) ter)
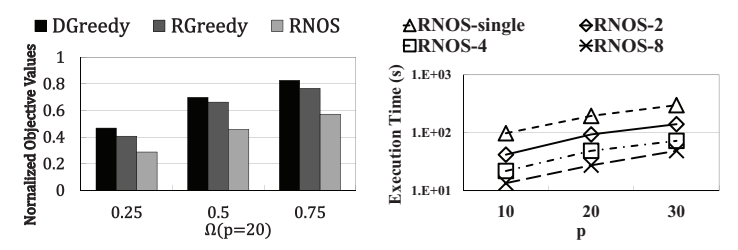

(c) Obj. values with diff. $\Omega$. (Twitter) (d) Execution time with diff. numbers of threads. (Twitter)
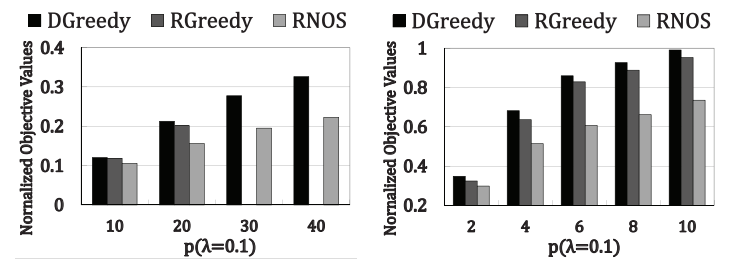

(e) Obj. values with diff. p. (Flickr) (f) Obj. values with diff. p. (Instagram)

Figure 3: Experimental results on large-scale datasets.

not return the solution for all users after 12 hours. The difference in execution time between RNOS and RGreedy becomes very significant as $p$ grows. The execution time of DGreedy and GBPR-TOP-p is the smallest since they only need to select the top- $p$ newsfeeds. Figure 3(b) with $\rho=0.3$ manifests that RNOS outperforms DGreedy, RGreedy, and GBPR-TOP-p in terms of normalized objective values (i.e., the addictive degree is divided by the maximum summation of addictive degrees), especially for a large $p$, because DGreedy does not consider the interplay between the addictive degrees and social support. On the other hand, RGreedy outperforms DGreedy since it has a chance to jump out of the local optimum by exchanging the newsfeeds. The results also indicate that highly addictive newsfeeds have more chances to be distributed by GBPR-TOP-p since it targets on preferences and is not designed to alleviate addiction.

Figure 3(c) compares the normalized objective values of DGreedy, RGreedy, and RNOS under different preference thresholds $\Omega$ with $p=20$. RNOS consistently outperforms DGreedy and RGreedy, especially for a large threshold. Notice that a tiny threshold usually generates a sudden change of contents and incurs anxiety [29]. Moreover, Figure 3(d) manifests that the processing can be boosted by around 6.3 times with $8 \mathrm{CPU}$ threads, and RNOS with parallelization is promising for deployment as a streaming cloud service. For Flickr and Instagram, RNOS significantly outperforms DGreedy and RGreedy as shown in Figures 3(e) and 3(f), while RGreedy does not return the results in 12 hours when $p \geq 30$. The number of newsfeeds (photos) is set as $2,4,6,8$, and 10 on Instagram since the dataset contains fewer photos. When $p$ increases, the objective 
values are greater on Instagram because many newsfeeds need to be selected to satisfy the size constraint.

\section{RELATED WORK}

Recent studies in Psychology and Sociology report several observations and offer various theories to explain the behaviors of addictive OSN users. Woods et al. find that SNAs frequently exhibit anxiety and low self-esteem [37]. Statistical analysis on addictions of mobile emails manifests that mobile email addiction may be a common phenomenon and associated with negative organizational consequences (e.g., turnover). Shuai et al. propose machine learning approaches to detect mental disorders [12]. However, the above research only studies the negative impacts of OSNs and does not provide any online intervention strategies.

To understand the semantics of newsfeeds, word embedding has drawn increasing attention to extract features from texts. One popular approach is Vector Space Model with TF-IDF weights. On the other hand, to find interesting newsfeeds for users, collaborative filtering [23] provides recommendations based on similar user preferences. Moreover, factorization and deep learning models are proposed to integrate auxiliary information for recommendation systems, e.g., RNN [7]. However, they do not carefully examine social interactions and leverage Psychology insights with benign and malicious envy to alleviate SNAs according to behavioral therapy, social support theory, and social comparison theory.

\section{CONCLUSION}

To the best of our knowledge, there exists no prior research that exploit data mining to alleviate SNAs while not sacrificing their preferences. In this paper, we first present $A D M$ that derives addictive degrees of newsfeeds, and then formulate $S A N S P$, prove its NP-Hardness, and design RNOS with a performance bound. A user study with 716 Facebook users and 11 mental healthcare professionals from hospitals around the world manifests that users show more than $30 \%$ of reduction on addictions with N3S, yet the usage time only slightly decreases (instead of plummeting as the abstinence approach).

\section{ACKNOWLEDGMENT}

This work is supported in part by NSF through grants IIS-1526499, IIS-1763325, and CNS-1626432, and NSFC 61672313, by Ministry of Science and Technology in Taiwan through grant 106-2221-E009-154-MY2, and by Academia Sinica in Taiwan through grant AS-106-TP-C05-3. Wang-Chien Lee's work is supported in part by the NSF under Grant No. IIS-1717084.

\section{REFERENCES}

[1] 2018. Newsfeed Filtering and Dissemination for Behavioral Therapy on Social Network Addictions. https://goo.gl/Sdk2HR. Technical report and datasets (2018)

[2] D. Agarwal and B.-C. Chen. 2009. Regression-based latent factor models. KDD.

[3] S. M. Alessi and N. M. Petry. 2003. Pathological gambling severity is associated with impulsivity in a delay discounting procedure. Behavioural Processes (2003).

[4] H. Appel, J. Crusius, and A. L. Gerla. 2015. Social comparison, envy, and depression on facebook: a study looking at the effects of high comparison standards on depressed individuals. Fournal of Social and Clinical Psychology (2015).

[5] Y. M. Baek, Y. Bae, and H. Jang. 2013. Social and parasocial relationships on social network sites and their differential relationships with users' psychological well-being. Cyberpsychology, Behavior, and Social Networking (2013).
[6] B. Bozoglan, V. Demirer, and I. Sahin. 2013. Loneliness, self-esteem, and life satisfaction as predictors of Internet addiction: a cross-sectional study among Turkish university students. Scandinavian fournal of Psychology (2013).

[7] A. Beutel et al. 2018. Latent cross: making use of context in recurrent recommender systems. In WSDM.

[8] A. Shensa et al. 2016. Social media use and perceived emotional support among US young adults. Fournal of Community Health (2016).

[9] B. K. et al. 1981. Effect of early and later colony housing on oral ingestion of morphine in rats. Pharmacology Biochemistry and Behavior (1981).

[10] C. Andreassen et al. 2012. Development of a Facebook addiction scale. Fournal of Psychology and Behavioral Sciences (2012).

[11] C. H. Chen et al. 2010. Efficient computation of optimal budget allocation for discrete event simulation experiment. IIE Transactions (2010).

[12] H.-H. Shuai et al. 2016. Mining online social data for detecting social network mental disorders. In $W W W$.

[13] L. Monacis et al. 2017. Social networking addiction, attachment style, and validation of the Italian version of the Bergen Social Media Addiction Scale. Fournal of Behavioral Addictions (2017).

[14] R. Li et al. 2012. Towards social user profiling: unified and discriminative influence model for inferring home locations. In $K D D$.

[15] S. Rendle et al. 2009. BPR: Bayesian personalized ranking from implicit feedback. In Conference on Uncertainty in Artificial Intelligence.

[16] T. Mikolov et al. 2013. Distributed representations of words and phrases and their compositionality. In NIPS.

[17] T. Ryan et al. 2014. The uses and abuses of Facebook: a review of Facebook addiction. Fournal of Behavioral Addictions (2014)

[18] E. Ferrara, R. Interdonato, and A. Tagarelli. 2014. Online popularity and topical interests through the lens of Instagram. In $H T$.

[19] R. He and J. McAuley. 2016. VBPR: Visual bayesian personalized ranking from implicit feedback. In $A A A I$

[20] C. J. Hutto and E. Gilbert. 2014. Vader: a parsimonious rule-based model for sentiment analysis of social media text. In ICWSM.

[21] H. H. Kim. 2016. The impact of online social networking on adolescent psychological well-being (WB): a population-level analysis of Korean school-aged children. International fournal of Adolescence and Youth (2016).

[22] I. Klerings, A. S. Weinhandl, and K. J. Thaler. 2015. Information overload in healthcare: too much of a good thing? Zeitschrift für Evidenz, Fortbildung und Qualität im Gesundheitswesen (2015).

[23] Y. Koren, R. Bell, and C. Volinsky. 2009. Matrix factorization techniques for recommender systems. IEEE Computer (2009).

[24] R. E. Korf. 1998. A complete anytime algorithm for number partitioning. Artificial Intelligence (1998).

[25] L.-W. Ku and H.-H. Chen. 2007. Mining opinions from the Web: beyond relevance retrieval. 7. Assoc. Inf. Sci. Technol. (2007).

[26] D. J. Kuss and M. D. Griffiths. 2011. Online social networking and addiction-a review of the psychological literature. International fournal of Environmental Research and Public Health (2011).

[27] A. B. Laudet, K. Morgen, and W. L. White. 2006. The role of social supports, spirituality, religiousness, life meaning and affiliation with 12-Step fellowships in quality of life satisfaction among individuals in recovery from alcohol and drug problems. Alcoholism Treatment Quarterly (2006)

[28] D. Meshi, C. Morawetz, and H. R. Heekeren. 2013. Nucleus accumbens response to gains in reputation for the self relative to gains for others predicts social media use. Frontiers in Human Neuroscience (2013).

[29] R. G. Miltenberger. 2011. Behavior modification: principles and procedures. Brooks/Cole Publishing Company.

[30] R. Moos. 2007. Theory-based active ingredients of effective treatments for substance use disorders. Drug and Alcohol Dependence (2007).

[31] G. OâĂŹKeeffe and K. Clarke-Pearson. 2011. Clinical report-the impact of social media on children, adolescents, and families. Pediatrics. Council on Communications and Media (2011).

[32] W. Pan and L. Chen. 2013. GBPR: group preference based bayesian personalized ranking for one-class collaborative filtering. In IJCAI.

[33] E. M. Perse and R. B. Rubin. 1989. Attribution in social and parasocial relationships. Communication Research (1989).

[34] R. Y. Rubinstein. 2001. Combinatorial optimization, cross-entropy, ants and rare events. In Stochastic Optimization: Algorithms and Applications.

[35] C. Wang, M. K. O. Lee, and Z. Hua. 2015. A theory of social media dependence: Evidence from microblog users. Decision Support Systems (2015).

[36] L. Weng, F. Menczer, and Y.-Y. Ahn. 2013. Virally prediction and community structure in social networks. Scientific Reports (2013).

[37] H. C. Woods and H. Scott. 2016. Sleepyteens: social media use in adolescence is associated with poor sleep quality, anxiety, depression and low self-esteem. Journal of Adolescence (2016).

[38] H.-H. Wu and W.-F. Lo. 2014. Why do you want to do "like", "comment" or "share" on Facebook: the study of antecedent on Facebook user's behavioral intentions. Marketing Review (2014). 